CARDIOVASCULAR MEDICINE

\title{
Relation between psychological strain and carotid atherosclerosis in a general population
}

\author{
B Wolff, H J Grabe, H Völzke, J Lüdemann, C Kessler, J B Dahm, H J Freyberger, U John, S B Felix
}

Heart 2005;91:460-464. doi: 10.1136/hrt.2003.031088

See end of article for authors' affiliations

\section{Correspondence to:} Dr Birger Wolff, Klinik für Innere Medizin B, ErnstMoritz-Arndt-Universität Greifswald, FriedrichLoeffler-Strasse $23 a$, 17487 Greifswald, Germany; birger.wolff@ uni-greifswald.de

Accepted 11 June 2004
Objective: To investigate the hypothesis that psychological strain is related to carotid atherosclerosis in a large general population sample.

Methods: Intima-media thickness and the prevalence of atherosclerotic plaques in the carotid arteries were quantitatively assessed by high resolution ultrasound among 2164 participants $(1112$ women and 1052 men, aged 45 to 75 years) of the SHIP (study of health in Pomerania), an epidemiological survey of a random sample of the population of north eastern Germany. Psychological strain was measured by 13 items reflecting typical psychological complaints. Each item was graded by the study participants on a four point scale (from 0, absent, to 3, severe) and a psychological strain score was generated by summing these 13 items.

Results: Mean psychological strain score was 10.8 (7.0) (median score 10) among women and 8.5 (6.2) (median score 8) among men. Psychological strain did not predict carotid intima-media thickness among either men or women. However, after adjustment for covariates, high psychological strain and carotid plaques were independently and linearly related, with plaque prevalence odds of $1.03(95 \%$ confidence interval (CI) 1.01 to $1.05, \mathrm{p}=0.009$ ) per increment of the psychological strain score among women and $1.04(95 \% \mathrm{Cl} 1.01$ to $1.07, \mathrm{p}=0.003)$ among men.

Conclusions: This study identified a relation between general psychological strain and carotid atherosclerosis.
S everal psychosocial factors such as depression, ${ }^{12}$ anxiety, ${ }^{3}$ chronic life stress, ${ }^{4}$ hopelessness, ${ }^{5}$ and certain behavioural patterns ${ }^{6}$ have been linked to the development of atherosclerosis. The underlying mechanisms are poorly understood. Increased sympathetic activation ${ }^{78}$ and decreased production of nitric oxide in response to mental stress $^{910}$ have been observed in some studies. Other investigations reported enhanced platelet activation, ${ }^{11}$ hypercortisolaemia, $^{12}$ and neuroendocrine activation ${ }^{13}{ }^{14}$ under conditions such as increased life stress and endogenous depression. Further possible explanations are a tendency to unhealthy lifestyle behaviours ${ }^{15}{ }^{16}$ and poor adherence to medical treatment among mentally ill patients. ${ }^{17}$ In this study, we investigate the association of self rated general psychological strain with carotid atherosclerosis in a random sample of the general population. Psychological strain was measured as a self rating score on 13 items that summarily comprise a broader spectrum of mental complaints.

\section{METHODS}

\section{Study population}

SHIP (the study of health in Pomerania) is a cross sectional study of the adult population of West Pomerania, the north eastern coastal region of Germany. From the total adult population of 212157 people living in the study area, a random sample from the population aged 20-79 years was drawn. The SHIP population comprised 4310 participants (68.8\% of eligible patients). The study was approved by the ethics committee of the University of Greifswald. All participants gave informed written consent. Data were collected between October 1997 and May 2001. Carotid arteries of 2344 participants (aged 45-75 years) were investigated by ultrasound. Study participants with a history of myocardial infarction or stroke as well as those with known malignancies were excluded. This resulted in a final sample of 2164 (1112 women, 1052 men) available for the current analysis. Sociodemographic characteristics and medical histories were assessed by computer aided face to face interviews. Hypertension was defined as a systolic blood pressure of $\geqslant 140 \mathrm{~mm} \mathrm{Hg}$, a diastolic blood pressure of $\geqslant 90 \mathrm{~mm} \mathrm{Hg}$, or self reported use of antihypertensive medications. Study participants were assigned to two smoking categories: smokers and non-smokers. Alcohol consumption was quantified in grams daily. Diabetes was defined as a self reported physician diagnosis of diabetes or serum haemoglobin $\mathrm{A}_{1 \mathrm{C}}$ of $>7.0 \%$. Height and weight were measured for calculation of the body mass index. A family history of coronary heart disease was considered to be positive if a parent or a sibling had ever sustained a fatal or non-fatal myocardial infarction. Haemoglobin $\mathrm{A}_{\mathrm{IC}}$ was determined by high performance liquid chromatography (DIAMAT; Bio-Rad Laboratories, Hercules, California, USA). High density lipoprotein (HDL) and low density lipoprotein (LDL) were measured photometrically (Boehringer Mannheim, Mannheim, Germany). Blood samples were analysed in a central laboratory that participated semi-annually in the official German tests for quality assurance. In addition, duplicate blood samples were collected and measured for internal quality assurance tests every week.

\section{Assessment of psychological strain}

All study participants completed a battery of psychosocial questionnaires. Part of these questionnaires was a modified form of von Zerssen's complaints scale, which has been used in a wide range of studies. ${ }^{18-20}$ These 13 items reflected typical psychological complaints and comprised the following symptoms: (1) sleep disturbances, (2) loss of energy, (3) weakness, (4) fatigue, (5) inner tension, (6) depression, (7)

Abbreviations: $\mathrm{Cl}$, confidence interval; $\mathrm{HDL}$, high density lipoprotein; IMT, intima-media thickness; LDL, low density lipoprotein; SHIP, the study of health in Pomerania 
rumination, (8) poor concentration, (9) anxiety, (10) agitation, (11) tremors, (12) nervousness, and (13) irritability. The study participants were asked to indicate to what degree they were affected by these complaints and to rate the presence of the respective complaint on a four point scale (absent, 0; mild, 1; moderate, 2; severe, 3). For the assessment of psychological distress a "strain score" was generated by summing the ratings of these 13 items. This strain score had an internal consistency of 0.89 (Cronbach's $\alpha$ ) and a Guttman split half reliability of 0.87 indicating high psychometric properties.

\section{Ultrasound measurements}

The ultrasound protocol has been previously described. ${ }^{21}$ In brief, certified medical assistants examined the extracranial carotid arteries bilaterally with B mode ultrasound with a $5 \mathrm{MHz}$ linear array transducer and a high resolution instrument (Diasonics VST Gateway, Santa Clara, California, USA). Both the near and far walls of the common carotid arteries, the internal carotid arteries, and the carotid bifurcations on both sides were evaluated online for the presence of atherosclerotic plaques. Each vessel segment was visualised in multiple longitudinal and transversal planes. Atherosclerotic plaques (yes/no) were defined by the following criteria: focal widening relative to adjacent segments (protrusion into the lumen or localised roughness with increased echogenicity); and an area of focal increased thickness $(\geqslant 1.3 \mathrm{~mm})$ of the intima-media layer. No attempts were made to further quantify plaque extension or severity. Plaque prevalence was defined as the presence of one or more plaques.

For measurement of carotid intima-media thickness (IMT), scans through the axis of the distal straight portion ( $1 \mathrm{~cm}$ in length) of both common carotid arteries were digitised and recorded for subsequent offline analysis. Certified readers calculated the mean far wall IMT by averaging 10 consecutive measurement points (in $1 \mathrm{~mm}$ steps) from both sides. The IMT was defined as the distance between the characteristic echoes from the lumen-intima and media-adventitia interfaces. Reproducibility between paired measurements of sonographers and readers has been studied. All measurements of intrareader, intrasonographer, interreader, and intersonographer variations had Spearman correlation coefficients of $>0.90$ and differences in mean $(2 \mathrm{SD})$ IMT of $<1.0 \%(<10.0 \%)$.

\section{Statistical analyses}

Groups were compared by Student's $t$ test for continuous variables and $\chi^{2}$ test for categorical variables. Multiple linear regression was used to analyse the association of the psychological strain score with carotid IMT. For this purpose, the psychological strain score was entered as a continuous variable into one model. In a further model, the 75th centile of the psychological strain score was used to dichotomise the study population into patients with high and those with low psychological strain. Data for women and men were analysed separately. The models were adjusted for covariates by inclusion of all of the following: age, hypertension, diabetes, smoking status, body mass index, alcohol consumption, family history of coronary heart disease, HDL, and LDL. Binary logistic regression with adjustment for the same covariates was used to calculate odds ratios and $95 \%$ confidence intervals (CI) with carotid plaque prevalence as the dependent variable and the psychological strain score as the independent variable of interest. A probability value of $\mathrm{p}<0.05$ was considered significant. All analyses were performed with SPSS software (version 11.0; SPSS Inc, Chicago, Illinois, USA).

\section{RESULTS}

Table 1 shows the clinical characteristics of all study participants. Men were older than women, had higher systolic and diastolic blood pressure, and accordingly were more likely to have hypertension. More men were current smokers and men consumed more alcohol than did women. Moreover, men had higher haemoglobin $\mathrm{A}_{1 \mathrm{C}}$ but lower HDL concentrations than women. Mean psychological strain score was 10.8 (7.0) (median score 10) among women and 8.5 (6.2) (median score 8$)$ among men $(\mathrm{p}<0.001$ for comparison between sexes). Figure 1 shows the distribution of psychological strain scores among women and men.

There was no association between psychological strain and carotid IMTs either among women or among men. These results remained unchanged after multivariate adjustment (data not shown). In further analyses, the 75th centile of the psychological strain score (score of 17 among women and 12 among men) was used to dichotomise the study population into patients with low and those with high psychological strain. However, there were no significant differences in adjusted carotid IMTs between patients with high and those with low psychological strain among either women (mean difference $0.004 \mathrm{~mm}, 95 \% \mathrm{CI}-0.015$ to 0.024 ) or men (mean difference $-0.003 \mathrm{~mm}, 95 \%$ CI -0.023 to 0.017$)$. Independent correlates of carotid IMT in our sample were age, sex, hypertension, diabetes, current smoking, HDL cholesterol, and LDL cholesterol (table 2).

A relation between the psychological strain score and the prevalence of carotid plaques was observed in the whole sample (age adjusted plaque prevalence odds per increment of the psychological strain score $1.03,95 \%$ CI 1.01 to 1.04 , $\mathrm{p}=0.002$ ) as well as among women (age adjusted plaque prevalence odds $1.03,95 \%$ CI 1.01 to $1.04, p=0.010$ ) and had borderline significance among men (plaque prevalence odds $1.03,95 \%$ CI 0.99 to $1.05, \mathrm{p}=0.056$ ).

Table 3 shows the relation between the psychological strain score and the prevalence of carotid plaques after multivariate adjustment. Among both women and men, psychological strain was independently associated with the prevalence of carotid plaques. When, assuming that psychological strain acts pathophysiologically similarly in both sexes, the female and male sample were combined, the same model yielded plaque prevalence odds of 1.04 (95\% CI 1.02 to 1.05 , $\mathrm{p}=0.001$ ) per increment in the psychological strain score. When the 75th centile of the psychological strain score was chosen to dichotomise the study population, high psychological strain was associated with plaque prevalence odds of

Table 1 Clinical characteristics of study participants

\begin{tabular}{|c|c|c|}
\hline & $\begin{array}{l}\text { Women } \\
(n=1112)\end{array}$ & $\begin{array}{l}\text { Men } \\
(n=1052)\end{array}$ \\
\hline $\begin{array}{l}\text { Age (years) } \\
\text { Systolic BP (mm Hg) } \\
\text { Diastolic BP (mm Hg) } \\
\text { Hypertension } \\
\text { Current smoking } \\
\text { Alcohol intake (g/day) } \\
\text { Diabetes } \\
\text { Haemoglobin } \mathrm{A}_{1 \mathrm{C}}(\%) \\
\text { BMI (kg/m²) } \\
\text { Family history of CHD } \\
\text { LDL (mmol/l) } \\
\text { HDL (mmol/I) } \\
\text { IMT (age adjusted) (mm) } \\
\text { Carotid plaque }\end{array}$ & $\begin{array}{l}59(8) \\
138(20) \\
84(10) \\
58.7 \% \\
17.9 \% \\
7.7(11.7) \\
10.6 \% \\
5.5(0.9) \\
28.5(5.2) \\
22.1 \% \\
3.9(1.1) \\
1.6(0.5) \\
0.74(0.01) \\
63.4 \%\end{array}$ & $\begin{array}{l}60(9)^{* *} \\
148(20)^{\star * *} \\
89(11)^{\star * *} \\
73.7 \%^{* * *} \\
27.5 \%^{* * *} \\
27.7(29.5)^{\star * *} \\
12.2 \% \\
5.7(1.0)^{\star *} \\
28.3(4.0) \\
19.9 \% \\
3.8(1.2) \\
1.3(0.4)^{* * *} \\
0.81(0.01)^{\star * *} \\
75.5 \%{ }^{*}\end{array}$ \\
\hline \multicolumn{3}{|c|}{$\begin{array}{l}\text { Continuous variables are mean (SD). } \\
{ }^{*} \mathrm{p}<0.05 ;{ }^{* *} \mathrm{p}<0.01 ;{ }^{* *} \mathrm{p}<0.001 \text { men } v \text { women. } \\
\mathrm{BMI} \text {, body mass index; BP, blood pressure; CHD, coronary heart disease; } \\
\mathrm{HDL} \text {, high density lipoprotein; IMT intima-media thickness; LDL, low } \\
\text { density lipoprotein. }\end{array}$} \\
\hline
\end{tabular}



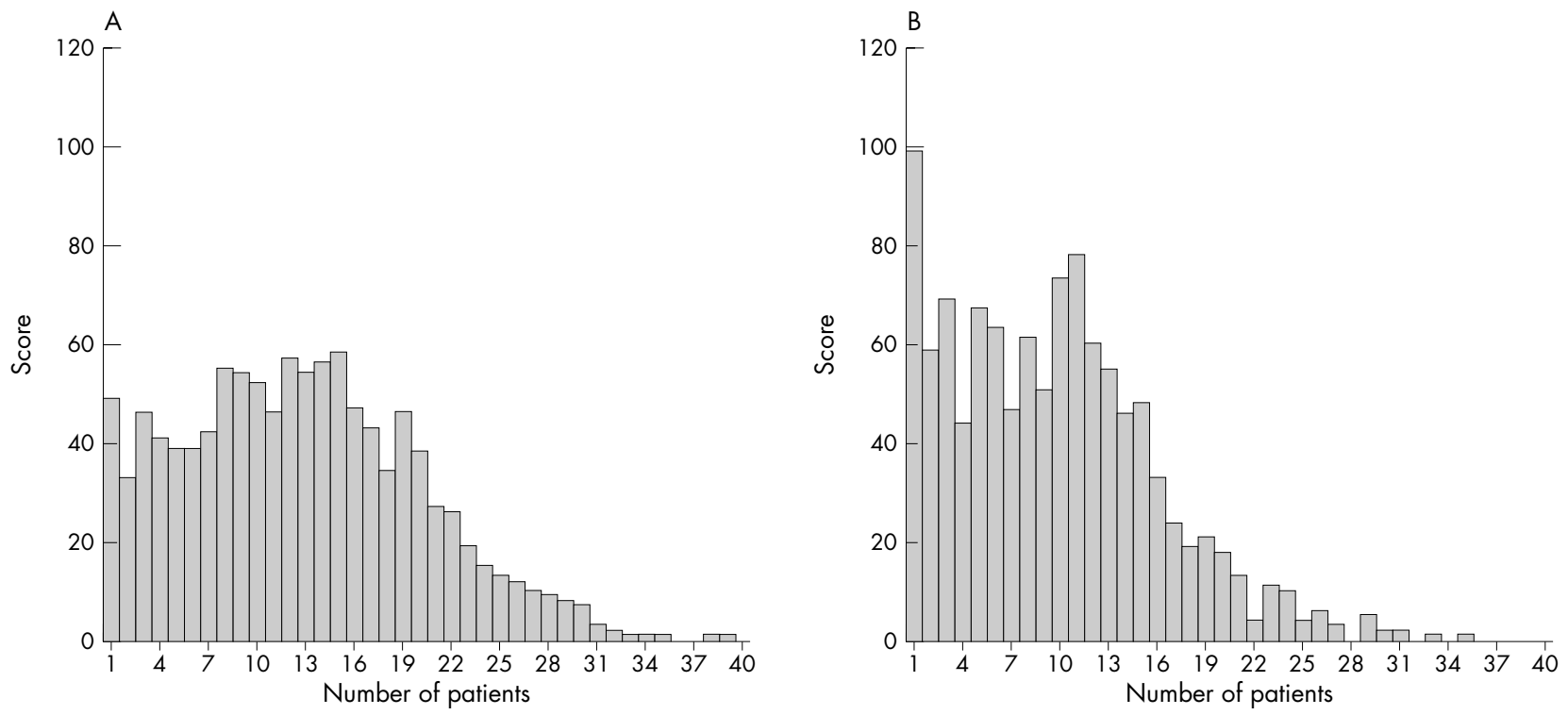

Figure 1 Distribution of general psychological strain as assess by 13 items reflecting typical mental complaints among (A) women and (B) men.

1.55 (95\% CI 1.10 to $2.18, \mathrm{p}=0.011)$ among women and 1.38 (95\% CI 1.12 to $1.75, \mathrm{p}=0.005$ ) among men.

Lastly, to rule out confounding of mental complaints by cerebral ischaemia, we excluded the 31 patients with a carotid stenosis of $\geqslant 50 \%$ ( 11 women, 20 men) in a further analysis. However, the above mentioned results remained unchanged after exclusion of patients with carotid stenosis.

\section{DISCUSSION}

In this study, we evaluated in a large general population sample of both sexes and covering a broad age range the relation between general psychological strain, as a measure of impaired mental well being, and carotid atherosclerosis. We observed a linear relation between self rated psychological strain and carotid plaque prevalence in both sexes.

Since the relation between carotid atherosclerosis and both coronary heart disease $\mathrm{e}^{22}$ and stroke ${ }^{24}$ is established, our findings may have several implications. Firstly, general psychological strain was assessed by 13 items related to common mental complaints of everyday life. Thus, our data discloses that findings of prior studies that described similar associations for symptoms of depression and anxiety can be extended to a more general measure of impaired mental well being. Secondly, our results further support the concept that behavioural interventions aimed at alleviating psychological stress may help reduce cardiovascular risk and that early commencement of primary prevention may be encouraged for psychologically strained patients. Early primary prevention was recently studied in hypertensive African Americans ${ }^{25}$ whose participation in a psychotherapeutic stress reduction programme was associated with reduced carotid atherosclerosis.

We could not establish an association of general psychological strain with carotid IMT. Relations between psychosocial variables and carotid IMT have been previously described. An investigation among middle aged postmenopausal women ${ }^{26}$ found associations between carotid IMT and the degree of anger suppression and hostility. However, unlike general psychological strain, anger suppression and hostility are more specific behavioural traits. In addition, these findings were obtained in a closely restricted study population, which may, at least in part, have accounted for these results. A further study that addressed the question of whether psychosocial stress is related to carotid atherosclerosis $^{27}$ likewise reported associations with plaque prevalence but not with carotid IMT. Moreover, although plaque formation and intima-media thickening are essential components of atherogenesis, they are pathologically distinct processes acting independently of each other. ${ }^{28}$ Whereas smooth muscle cell proliferation and lipid deposition predominate in intima-media thickening, plaque formation and growth largely rely on factors that promote thrombosis, hypercoagulability, and attenuated fibrinolysis. ${ }^{29}$ Previous studies showed that mental disorders are associated with

Table 2 Relation between cardiovascular risk factors and IMT

\begin{tabular}{|c|c|c|c|c|c|c|}
\hline & \multicolumn{3}{|l|}{ Women } & \multicolumn{3}{|l|}{ Men } \\
\hline & B & $95 \% \mathrm{Cl}$ & $\beta$ & B & $95 \% \mathrm{Cl}$ & $\beta$ \\
\hline $\begin{array}{l}\text { Age per } 1 \text { year increase } \\
\text { Hypertension } \\
\text { Diabetes } \\
\text { Current smoking } \\
\text { HDL per increase by } 1.0 \mathrm{mmol} / / \\
\text { LDL per increase by } 1.0 \mathrm{mmol} / \mathrm{I}\end{array}$ & $\begin{array}{r}0.0057 \\
0.0548 \\
0.0571 \\
0.0071 \\
-0.0267 \\
0.0109\end{array}$ & $\begin{array}{l}0.005 \text { to } 0.007^{* * *} \\
0.036 \text { to } 0.073^{* * *} \\
0.028 \text { to } 0.083^{* * *} \\
0.001 \text { to } 0.002^{*} \\
-0.045 \text { to }-0.008^{* *} \\
0.003 \text { to } 0.019^{* *}\end{array}$ & $\begin{array}{r}0.304 \\
0.177 \\
0.114 \\
0.067 \\
-0.083 \\
0.078\end{array}$ & $\begin{array}{r}0.0081 \\
0.0422 \\
0.0322 \\
0.0231 \\
-0.0332 \\
0.0160\end{array}$ & $\begin{array}{l}0.007 \text { to } 0.009^{* * *} \\
0.021 \text { to } 0.046^{* * *} \\
0.005 \text { to } 0.061^{*} \\
0.015 \text { to } 0.032^{* * *} \\
-0.057 \text { to }-0.009^{* *} \\
0.008 \text { to } 0.024^{* * *}\end{array}$ & $\begin{array}{r}0.421 \\
0.113 \\
0.069 \\
0.155 \\
-0.077 \\
0.117\end{array}$ \\
\hline
\end{tabular}


Table 3 Odds ratios (OR) for prevalence of atherosclerotic plaques associated with cardiovascular risk factors

\begin{tabular}{|c|c|c|c|c|c|c|}
\hline & \multicolumn{3}{|c|}{ Women } & \multicolumn{3}{|l|}{ Men } \\
\hline & $\overline{O R}$ & $95 \% \mathrm{Cl}$ & Wald & $\overline{O R}$ & $95 \% \mathrm{Cl}$ & Wald \\
\hline Psychological strain per increment & 1.03 & 1.01 to $1.05^{\star *}$ & 6.18 & 1.04 & 1.01 to $1.07^{\star *}$ & 6.66 \\
\hline Age per 1 year increase & 1.14 & 1.12 to $1.17^{\star \star \star *}$ & 151.21 & 1.11 & 1.09 to $1.14^{\star \star *}$ & 84.82 \\
\hline Hypertension & 1.58 & 1.17 to $2.13^{\star *}$ & 8.92 & 1.95 & 1.39 to $2.74^{\star \star *}$ & 11.41 \\
\hline Diabetes & 1.44 & 0.98 to 2.45 & 3.12 & 1.98 & 1.14 to $3.39^{*}$ & 5.96 \\
\hline Current smoking & 1.18 & 1.06 to $1.34^{* *}$ & 6.53 & 1.24 & 1.07 to $1.44^{* *}$ & 8.33 \\
\hline BMI per increase by 1.0 & 1.06 & 1.03 to $1.09^{* * *}$ & 15.53 & 1.03 & 1.00 to $1.08^{*}$ & 3.82 \\
\hline
\end{tabular}

${ }^{*} \mathrm{p}<0.05 ;{ }^{* *} \mathrm{p}<0.01 ;{ }^{* * *} \mathrm{p}<0.001$.

$\mathrm{OR}, 95 \% \mathrm{Cl}$, and Wald statistics are derived from binary logistic regression models with plaque prevalence as dependent variable and all of the following as independent variables: psychological strain, age, hypertension, diabetes, smoking status, alcohol consumption, HDL, LDL, BMI, and family history of CHD.

enhanced platelet reactivity and increased plasma concentrations of several prothrombotic factors. ${ }^{11}{ }^{30-32}$ Thus, although our study provides no evidence for a pathobiochemical explanation, enhanced platelet function and increased prothrombotic capacity may, on the one hand, be mechanisms by which psychological strain promotes plaque formation. On the other hand, further mechanisms, such as cross talk between mental stress and blood pressure, may also be involved. Mental stress can produce rapid sympathetic activation, ${ }^{33}$ which translates into changes in systemic haemodynamic function. ${ }^{34}$ Sustained psychosocial and vocational stress has been associated with ambulatory blood pressure in British teachers. ${ }^{35}$ Furthermore, it was shown that people with higher rises in systolic blood pressure during frustrating psychological stress have more severe ${ }^{36}$ and greater progression ${ }^{37}$ of carotid atherosclerosis. Another study described that, in otherwise healthy young people, even brief episodes of mental stress can elicit transient endothelial dysfunction. ${ }^{10}$ Assuming that psychologically strained people have more severe and more frequent mental stress, they may, as a consequence, experience repetitive and prolonged periods of endothelial dysfunction. The relation between mental stress and endothelial dysfunction may therefore constitute a further link between psychological strain and carotid atherosclerosis.

Some limitations of our study merit discussion. The cross sectional nature of our data does not allow us to assess the time sequence of the relation between psychological strain and carotid atherosclerosis. Moreover, the severity of psychological strain may fluctuate such that the measured severity probably does not accurately reflect the course over the time in which carotid atherosclerosis develops. This limitation, though, becomes negligible by chance in a sufficiently large number of study participants. Moreover, we had no information on the duration and the course (episodic versus chronic) of psychological complaints. Further studies should examine whether these temporal characteristics have an impact on the association between psychological strain and atherosclerosis.

In conclusion, we identified a linear relation between general psychological strain and carotid atherosclerosis. This relation was established for carotid plaque prevalence but not for carotid IMT. This circumstance may be attributable to interference of psychological strain with cardiovascular risk factors that are preferentially involved in plaque formation and growth. Since high psychological strain is a potentially modifiable and reversible condition, our findings may have implications for the treatment and prevention of atherosclerosis.

\section{ACKNOWLEDGEMENTS}

The SHIP study is part of the Community Medicine Net (http:// www.medizin.uni-greifswald.de/(cm) of the University of Greifswald, which is funded by grants from the German Federal Ministry of Education and Research (BMBF, grant 01ZZ96030) and the Ministry for Education, Research, and Cultural Affairs and the Ministry for Social Affairs of the Federal State of Mecklenburg-West Pomerania. Data collection contributed by the field workers, study physicians, ultrasound technicians, interviewers, and computer assistants is gratefully acknowledged.

\section{Authors' affiliations}

B Wolff, J B Dahm, S B Felix, Klinik für Innere Medizin B, Universität Greifswald, Greifswald, Germany

H Völzke, U John, Institut für Epidemiologie und Sozialmedizin, Universität Greifswald

J Lüdemann, Institut für Klinische Chemie, Universität Greifswald C Kessler, Klinik für Neurologie, Universität Greifswald

H J Grabe, H J Freyberger, Klinik für Psychiatrie und Psychotherapie, Universität Greifswald, Klinikum der Hansestadt Stralsund, Stralsund,

Germany

The funding source had neither been involved in the submission of the manuscript nor in the decision to publish the data. None of the authors has to make any financial disclosures or has a potential conflict of interest.

\section{REFERENCES}

1 Pratt LA, Ford DE, Crum RM, et al. Depression, psychotropic medication, and risk of myocardial infarction: prospective data from the Baltimore ECA followup. Circulation 1996;94:3123-9.

2 Barefoot J, Schroll M. Symptoms of depression, acute myocardial infarction, and total mortality in a community sample. Circulation 1996:93:1976-80.

3 Kawachi I, Colditz GA, Ascherio A, et al. Prospective study of phobic anxiety and risk of coronary heart disease in men. Circulation 1994;89:1992-7.

4 Rozanski A, Bairey CN, Krantz DS, et al. Mental stress and the induction of silent myocardial ischemia in patients with coronary artery disease. N Engl J Med 1988;318:1005-12.

5 Everson SA, Kaplan GA, Goldberg DE, et al. Hopelessness and 4-year progression of carotid atherosclerosis. The Kuopio ischemic heart disease risk factor study. Arterioscler Thromb Vasc Biol 1997; 17:1490-5.

6 Denollet J, Brutsaert DL. Personality, disease severity, and the risk of long term cardiac events in patients with a decreased ejection fraction after myocardial infarction. Circulation 1998;97:167-73.

7 Veith RC, Lewis L, Linares OA, et al. Sympathetic nervous system in major depression: basal and desipramine-induced alterations in plasma norepinephrine kinetics. Arch Gen Psychiatry 1994;51:411-22.

8 Dakak N, Quyyumi AA, Eisenhofer G, et al. Sympathetically mediated effects of mental stress on the cardiac microcirculation of patients with coronary artery disease. Am J Cardiol 1995;76:125-30.

9 Cardillo C, Kilcoyne CM, Cannon RO 3rd, et al. mpairment of the nitric oxidemediated vasodilator response to mental stress in hypertensive but not in hypercholesterolemic patients. J Am Coll Cardiol 1998;32:1207-13.

10 Ghiadoni L, Donald AE, Cropley M, et al. Mental stress induces transient endothelial dysfunction in humans. Circulation 2000;102:2473-8.

11 Musselman DL, Tomer A, Manatunga AK, et al. Exaggerated platelet reactivity in major depression. Am J Psychiatry 1996;153:1313-7.

12 Gold PW, Loriaux DL, Roy A, et al. Response to corticotropin releasing hormone in the hypercortisolism of depression and Cushing's disease. N Engl J Med 1986;314:1329-35.

13 Nemeroff CB, Widerlov E, Bissette G, et al. Elevated concentrations of CSF corticotropin releasing factor-like immunoreactivity in depressed patients. Science 1984;226:1342-4.

14 Suarez EC, Kuhn CM, Schanberg SM, et al. Neuroendocrine, cardiovascular, and emotional responses of hostile men: the role of interpersonal challenge. Psychosom Med 1998;60:78-88

15 Glassman AH, Helzer JE, Covey LS, et al. Smoking, smoking cessation and major depression. JAMA 1990;264:1546-9.

16 Ziegelstein RC, Bush DE, Fauerbach JA. Depression, adherence behavior, and coronary disease outcomes. Arch Intern Med 1998;158:808-9. 
17 Carney RM, Freedland KE, Eisen SA, et al. Major depression and medication adherence in elderly patients with coronary artery disease. Health Psychol 1995; 14:88-90.

18 Von Zerssen D, Koeller D. Beschwerden-Liste [complaints schedule]. Weinheim: Beltz, 1976.

19 Baver M, Priebe S, Berghofer A, et al. Subjective response to and tolerability of long-term supraphysiological doses of levothyroxine in refractory mood disorders. J Affect Disord 2001;64:35-42.

20 Baltes PB, Mayer KU, eds. The Berlin aging study: aging from 70 to 100 . New York: Cambridge University Press, 1999.

21 Luedemann J, Schminke U, Berger K, et al. Association between behaviordependent cardiovascular risk factors and asymptomatic carotid atherosclerosis in a general population. Stroke 2002;33:2929-35.

22 Craven TE, Ryu JE, Espeland MA, et al. Evaluation of the associations between carotid artery atherosclerosis and coronary artery stenosis: a case-control study. Circulation 1990;82:1230-42.

23 Kallikazaros I, Tsioufis C, Sideris S, et al. Carotid artery disease as a marker for the presence of severe coronary artery disease in patients evaluated for chest pain. Circulation 1999:30:1002-7.

24 Gronholdt ML, Nordestgaard BG, Schroeder TV, et al. Ultrasonic echolucent carotid plaques predict future strokes. Circulation 2001;104:68-73.

25 Castillo-Richmond A, Schneider RH, Alexander CN, et al. Effects of stress reduction on carotid atherosclerosis in hypertensive African Americans. Stroke 2000;31:568-73.

26 Matthews KA, Owens JF, Kuller LH, et al. Are hostility and anxiety associated with carotid atherosclerosis in healthy postmenopausal women? Psychosom Med 1998;60:633-8.
27 Rosvall M, Ostergren PO, Hedblad B, et al. Work-related psychosocial factors and carotid atherosclerosis. Int J Epidemiol 2002;31:1169-78.

28 Ross R, Glomset JA. The pathogenesis of atherosclerosis. N Engl J Med 1976;295:369-77.

29 Willeit J, Kiechl S, Oberhollenzer F, et al. Distinct risk profiles of early and advanced atherosclerosis. Arterioscler Thromb Vasc Biol 2000;20:529-37.

30 Laghrissi-Thode F, Wagner WR, Pollock BG, et al. Elevated platelet factor 4 and b-thromboglobulin plasma levels in depressed patients with ischemic heart disease. Biol Psychiatry 1997:42:290-5.

31 Ogston D, McDonald GA, Fullerton HW. The influence of anxiety on tests of blood coagulation and fibrinolytic activity. Lancet, 1962;ii, 521-3.

32 Levine SP, Towell BL, Suarez AM, et al. Platelet activation and secretion associated with emotional stress. Circulation 1985;71:1129-34.

33 Noll G. Wenzel RR, Schneider M et al. Increased activation of sympathetic nervous system and endothelin by mental stress in normotensive offspring of hypertensive parents. Circulation 1996;93:866-9.

34 Steptoe A, Fieldman G, Evans O, et al. Cardiovascular risk and responsivity to mental stress: the influence of age, gender and risk factors. J Cardiovasc Risk 1996:3:83-93

35 Steptoe A, Cropley M. Persistent high job demands and reactivity to mental stress predict future ambulatory blood pressure. J Hypertens 2000;18:581-6.

36 Kamarck TW, Everson SA, Kaplan GA, et al. Exaggerated blood pressure responses during mental stress are associated with enhanced carotid atherosclerosis in middle-aged Finnish men: findings from the Kuopio ischemic heart disease study. Circulation 1997;96:3842-8.

37 Barnett PA, Spence JD, Manuck SB, et al. Psychological stress and the progression of carotid artery disease. J Hypertens 1997;15:49-55.

\section{IMAGES IN CARDIOLOGY}

\section{Non-invasive follow up of an atrial septal defect device occlusion}

A n asymptomatic 13 year old girl was found to have a fixed and widely split second heart sound on a routine examination. There was also a grade $2 / 6$ systolic ejection murmur heard best at the left upper sternal border. The ECG showed right atrial and ventricular enlargement with right axis deviation. The chest $x$ ray revealed mild cardiomegaly with increased pulmonary blood flow. Transthoracic echocardiography displayed a $16 \mathrm{~mm}$ secundum type atrial septal defect (ASD) with a Qp:Qs of $>$ 4.0:1.0. The defect was closed percutaneously with a $24 \mathrm{~mm}$ Amplatzer ASD occluding device. Transthoracic echocardiography (panel A) and cardiac magnetic resonance

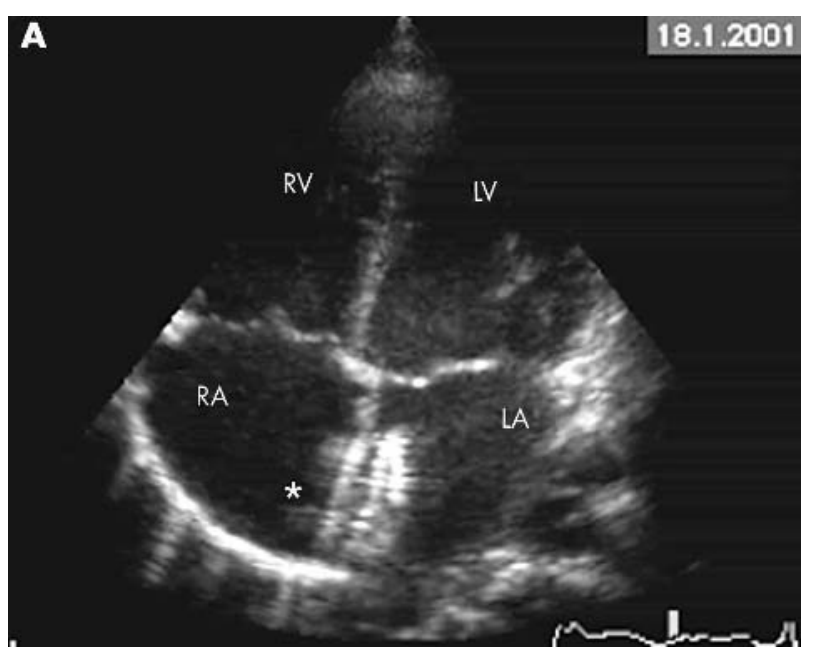

Echocardiography, apical four chamber view. LA, left atrium, LV, left ventricle; RA, right atrium; RV right ventricle; * occluding device. imaging (panel B) non-invasively confirmed the appropriate placement of the device in regard to the atrial septum and adjacent cardiac structures. No residual shunt, impairment of the atrioventricular valves, or obstruction of the coronary sinus was found. The post-interventional course was uneventful. The observation period is 11 months. Transoesophageal imaging seems avoidable for routine follow up.

\section{Lilje \\ F Weiss \\ J Weil}

lilje@europe.com

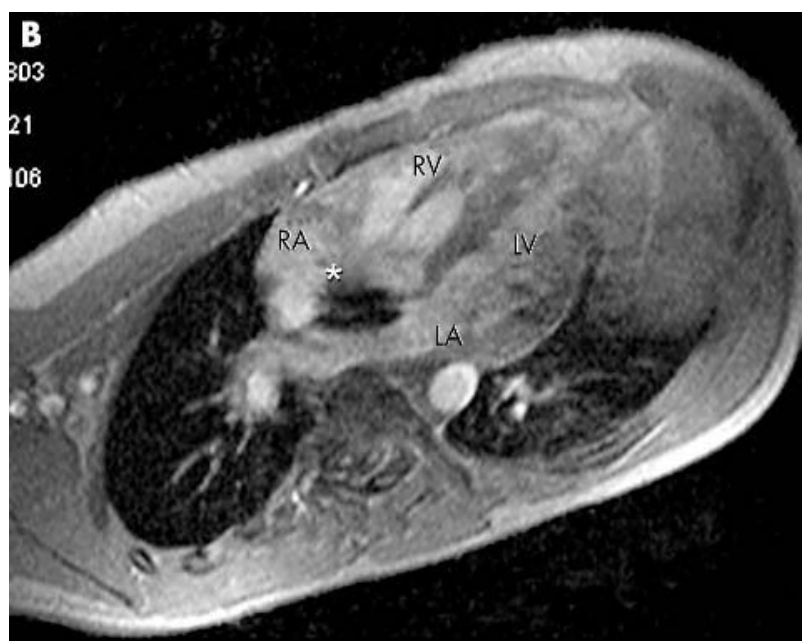

Magnetic resonance imaging, coronal scan, $T_{1}$ weighted spoiled gradient echo (FLASH). The device is properly located. Both the left and right atrial discs are clearly visualised. 